\title{
ON THE UNIFORM ERGODIC THEOREM
}

\author{
MICHAEL LIN ${ }^{1}$
}

ABSTRACT. We give an elementary proof of the uniform ergodic theorem: "Let $T$ be a linear operator on a Banach space with $\left\|T^{n} / n\right\| \rightarrow 0$. The following are equivalent: (1) $N^{-1} \sum_{n=0}^{N-1} T^{n}$ converges uniformly. (2) $(I-T)^{2} X$ is closed. (3) $(I-T) X$ is closed."

In 1943, N. Dunford [1] obtained certain ergodic theorems as corollaries of the operational calculus for analytic functions of an operator on a complex Banach space. One of these results is given in the equivalence of the first three conditions of the following theorem. The equivalence of condition (4) seems to have been unnoticed by Dunford, since in [2, p. 649] he remarks that the mean ergodic theorem has to be assumed to prove sufficiency.

The proof given here does not depend on the spectral analysis and applies to real or complex Banach spaces. Some corollaries are given.

THEOREM. Let $T$ be a bounded linear operator on a Banach space $X$ satisfying $\left\|T^{n} / n\right\| \rightarrow 0$. Then the following conditions are equivalent:

(1) There exists a bounded linear operator $E$ such that

$$
\left\|N^{-1} \sum_{n=0}^{N-1} T^{n}-E\right\| \rightarrow 0 .
$$

(2) $(I-T) X$ is closed and $X=\{x: T x=x\} \oplus(I-T) X$.

(3) $(I-T)^{2} X$ is closed.

(4) $(I-T) X$ is closed.

Proof. We denote $Y=\mathrm{Cl}((I-T) X)$. (1) $\Rightarrow(2)$ : By (1) we necessarily have $E^{2}=E$ with $E X=\{x: T x=x\}$, and $X=E X \oplus Y$. $Y$ is invariant under $T$, and the restriction $S=T_{\mid Y}$ satisfies $\left\|N^{-1} \sum_{n=0}^{N-1} S^{n}\right\| \rightarrow 0$. Fix $N$ such that $\left\|N^{-1} \sum_{n=0}^{N-1} S^{n}\right\|<1$. Then $I-N^{-1} \sum_{n=0}^{N-1} S^{n}$ is invertible, and so is $I-S$; thus $Y=(I-S) Y=(I-T) Y \subset(I-T) X$, and $Y=(I-T) X$.

$(2) \Rightarrow(3)$ : We have $Y=(I-T) X$ and $(I-T)^{2} X \subset Y$. Let $y \in Y$. Then $y=(I-T) x$. Since $x=x_{0}+x_{1}$ with $T x_{0}=x_{0}$ and $x_{1} \in Y$, we have $y=$ $(I-T) x=(I-T) x_{1} \in(I-T) Y=(I-T)^{2} X$, and $(I-T)^{2} X=Y$ is closed.

Received by the editors May 7, 1973.

AMS (MOS) subject classifications (1970). Primary 47A35, 28A65; Secondary $60 \mathrm{~J} 05,54 \mathrm{H} 20$.

Key words and phrases. Ergodic theorem, quasi-compact operators, Markov operators.

${ }^{1}$ Research partly supported by NSF Grant GP 34118.

(c) American Mathematical Society 1974 
(3) $\Rightarrow(4):(I-T) Y=(I-T)^{2} X$ is easily checked, by (3). The restriction $S=T_{\mid Y}$ satisfies $\left\|N^{-1} \sum_{n=0}^{N-1} S^{n} y\right\| \rightarrow 0$ for $y \in(I-T) X$, so that

$$
(I-T) X \subset \mathrm{Cl}((I-S) Y)=(I-S) Y=(I-T)^{2} X .
$$

Thus $Y \subset(I-T)^{2} X \subset(I-T) X$ and $(I-T) X$ is closed.

(4) $\Rightarrow(1)$ : By the open mapping theorem there exists a $K>0$ such that for $y \in(I-T) X \equiv Y$ there is an $x \in X$ with $(I-T) x=y$ and $\|x\| \leqq K\|y\|$ [3, p. 487]. Thus for $y \in Y$ we have

$$
\left\|N^{-1} \sum_{n=0}^{N-1} T^{n} y\right\|=\left\|N^{-1} \sum_{n=0}^{N-1} T^{n}(I-T) x\right\| \leqq\left\|\frac{I-T^{N}}{N}\right\| K\|y\|
$$

so that $S$, the restriction of $T$ to $Y$, satisfies $\left\|N^{-1} \sum_{n=0}^{N-1} S^{n}\right\| \rightarrow 0$ and by the proof of $(1) \Rightarrow(2) I-S$ is invertible on $Y$ and $(I-T) X=Y=(I-S) Y=$ $(I-T) Y=(I-T)^{2} X$. Hence for $x \in X$ there is $y \in Y$ with $(I-T) x=$ $(I-T) y$. Thus $x=(x-y)+y$ and $X=\{x: T x=x\} \oplus(I-T) X$. This shows that $N^{-1} \sum_{n=0}^{N-1} T^{n}$ converges strongly, and $E=\lim N^{-1} \sum_{n=0}^{N-1} T^{n}$ is a bounded projection on $\{x: T x=x\}$, and its null-space is $(I-T) X$ (which is closed). (1) follows because $(I-T)$ is invertible on $Y$.

REMARK. We can also add the condition " $(I-T) X$ is closed" to Theorem 3.16 of [1]. Since its necessity is proved in [1], we sketch the proof of its sufficiency using the ideas of the previous proof (the notation is that of [1]): We may and do assume that $f_{n}(1)=1$, by looking at $f_{n}(z) / f_{n}(1)$, since $f_{n}(1) \rightarrow 1$. Thus $f_{n}(1)-1=0$, so that we can find analytic functions $g_{n}(z)$ such that $g_{n}(z)(1-z)=f_{n}(z)-1$ (with $g_{n}$ defined where $f_{n}$ is defined). On $Y=(I-T) X$ we can show $\left\|f_{n}(T)\right\| \rightarrow 0$ so that $I-f_{n}(T)$ is invertible on $Y$ and therefore $(I-T) g_{n}(T)$ is invertible on $Y$ and so is $I-T$. Hence $(I-T)^{2} X=(I-T) Y$ is closed, and this is one of the equivalent conditions in [1].

Corollary 1. Let T satisfy $\left\|T^{n} / n\right\| \rightarrow 0$. (1) If $\sup _{N}\left\|\sum_{n=0}^{N} T^{n} y\right\|<\infty$ for every $y \in \mathrm{Cl}((I-T) X)$, then $N^{-1} \sum_{n=0}^{N-1} T^{n}$ converges uniformly. (2) If $\sup _{n}\left\|T^{n}\right\|<\infty$, the converse of (1) is also true.

Proof. (1) $Y=\mathrm{Cl}((I-T) X)$ is invariant under $T$, and $S=T_{\mid Y}$ satisfies $\left\|S^{n}\right\| \leqq\left\|T^{n}\right\|$. By the principle of uniform boundedness there exists a $K>0$ such that $\sup _{N}\left\|\sum_{n=0}^{N} S\right\|<K$, and $\left\|N^{-1} \sum_{n=0}^{N-1} S^{n}\right\| \rightarrow 0$ and by the theorem $Y=(I-S) Y \subset(I-T) X$. Thus $(I-T) X$ is closed and we apply the theorem again. (2) If $N^{-1} \sum_{n=0}^{N-1} T^{n}$ converges uniformly, $(I-T) X$ is closed and $\sup _{N}\left\|\sum_{n=0}^{N} T^{n} y\right\|<\infty$ for $y \in(I-T) X$.

COROLLARY 2. Assume sup $\left\|T^{n}\right\|<\infty$. If there exist nonnegative numbers $a_{1}, \cdots, a_{k}$ with $\sum_{i=1}^{k} a_{i}=1$ such that $\left\|\sum_{i=1}^{k} a_{i} T^{i}-Q\right\|<1$ for some compact 
operator $Q$, then $N^{-1} \sum_{n=0}^{N-1} T^{n}$ converges uniformly (to a finite-dimensional projection).

Proof. Let $A=\sum_{i=1}^{k} a_{i} T^{i}$. It is easy to see that $\sup \left\|A^{n}\right\|<\infty$. To prove that $(I-A) X$ is closed, we show $(I-A) Y=Y$, where

$$
Y=\mathrm{Cl}((I-A) X) \text {. }
$$

Denote $V=A-Q$. If $y=\lim y_{n}$ with $y_{n} \in(I-A) Y$, we show that $y \in$ $(I-A) Y$ similarly to the (simple) proof of Lemma VII.4.1 in [3], using the fact that $I-V$ is invertible. By the theorem we have that $I-A$ is invertible on its (closed) range $Y$, and $X=\{x: A x=x\} \oplus Y$. But

$$
I-A=\sum_{i=1}^{k} a_{i}\left(I-T^{i}\right)=(I-T) \sum_{i=1}^{k} a_{i}\left(\sum_{j=0}^{i-1} T^{j}\right)
$$

and since $T Y \subset Y, I-T$ is invertible on $Y . Z=\{x: A x=x\}$ is finite dimensional (since $\|A-Q\|<1$ ) and $T$-invariant. From this we have easily that $\left\|N^{-1} \sum_{n=0}^{N-1} T^{n}-E\right\| \rightarrow 0$, the limit $E$ being a projection on a subspace of $Z$.

RemarK. This result, when $a_{k}=1$ ( $T$ quasi-compact), is due to Yosida and Kakutani [8], who obtained the spectral analysis (on a complex Banach space) of $T$ and deduced the corollary. A proof, in their set-up, which looks only at eigenvalues of unit modulus, is given in Loève [6]. Another proof for Markov operators is given in Neveu [7]. The assumption sup $\left\|T^{n}\right\|<\infty$ is used to insure that $\operatorname{Cl}((I-A) X)$ does not contain fixed points of $A$. The methods of Dunford and Schwartz [3], using spectral analysis and the operational calculus, prove the result of [8] with the assumption $T^{n} / n \rightarrow 0$ in the weak operator topology. Their method can prove Corollary 2 when $\left\|T^{n} / n\right\| \rightarrow 0$. (If $A=\sum a_{i} T^{i}$, then $|\sigma(A)| \leqq 1$ since $|\sigma(T)| \leqq 1$. By Lemma VIII.8.2 of [3] the spectral points of $A$ of unit modulus are isolated with corresponding finite-dimensional projections. Then $X=X_{1} \oplus X_{2}$ where, on $X_{1},\left\|N^{-1} \sum_{n=0}^{N-1} A^{n}\right\| \rightarrow 0$, and $X_{2}$ is finite dimensional, both invariant under $A$, given by spectral projections of $A$. Hence by Theorem VII.3.19 of [3] $X_{1}$ and $X_{2}$ are invariant under T. On $X_{1}$ the uniform convergence to 0 follows because $I-T$ is invertible there, and on $X_{2} T$ is compact and we apply Theorem VIII.8.3. The real case can be deduced from the complex one.)

CoRollary 3. Let $T$ satisfy $\left\|T^{n} / n\right\| \rightarrow 0$ and let $Z \subset X^{*}$ be a closed subspace invariant under $T^{*}$. If $(I-T) X$ is closed, $\left(I-T^{*}\right) Z$ is closed.

EXAMPLE. Corollary 3 can be applied to the operators induced by a Markov transition probability, with $X$ being either the space of bounded measurable functions or finite signed measures, and $Z$ the other space.

The results are now applied to a problem in topological dynamics. 
Proposition. Let $S$ be a (nondiscrete) compact metrizable space, and let $\theta$ be a continuous mapping of $S$ into itself having a unique invariant probability, $\lambda$. Assume that $\lambda(U)>0$ for $\varnothing \neq U$ open. Then there exists a continuous function $f$ with $\int f d \lambda=0$ such that $\sup _{N}\left\|\sum_{n=0}^{N} f\left(\theta^{n} x\right)\right\|=\infty$.

Proof. Let $T$ be the operator on $C(S)$ defined by $T f(x)=f(\theta x)$. By assumption, $\operatorname{Cl}((I-T) C(S))=\left\{f \in C(S): \int f d \lambda=0\right\}$. If the assertion fails, Corollary 1 shows that $N^{-1} \sum_{n=0}^{N-1} T^{n}$ converges uniformly, and $N^{-1} \sum_{n=0}^{N-1} T^{* n}$ also converges uniformly (on the space of finite signed Borel measures). Let $P$ be the Markov operator on $L_{1}(\lambda)$ defined by $u P=d\left(T^{*} \mu\right) / d \lambda$ when $u=d \mu / d \lambda$. Then $N^{-1} \sum_{n=0}^{N-1} P^{n}$ also converges uniformly, and $P$ is conservative and ergodic; hence by Horowitz [5] $P$ is Harris. But the Borel field is not atomic modulo $\lambda$ ( $S$ is not discrete) contradicting the atomicity of the deterministic field of a Harris process. Hence the assertion is true.

Remarks. (1) Gottschalk and Hedlund [4, p. 138] give a method of constructing such functions $f$ in a particular situation. (2) Horowitz [5] also proved that for a conservative and ergodic Markov operator the uniform ergodic theorem is equivalent to the strong ergodic theorem (in $L_{\infty}$ ). The proposition shows that the analogue for an operator $T$ on $C(S)$ fails, if $P$ is not Harris.

Acknowledgement. I am grateful to Professor William B. Johnson for detecting an omission in my first proof of the theorem. Professor T. Figiel has remarked that all that is needed in Corollary 2 is that $I-\sum_{i=1}^{k} a_{i} T^{i}+Q$ be invertible.

\section{REFERENCES}

1. N. Dunford, Spectral theory. I. Convergence to projections, Trans. Amer. Math. Soc. 54 (1943), 185-217. MR 5, 39.

2. —, Spectral theory, Bull. Amer. Math. Soc. 49 (1943), 637-651. MR 5, 39.

3. N. Dunford and J. T. Schwartz, Linear operators. I: General theory, Pure and Appl. Math., vol. 7, Interscience, New York, 1958. MR 22 \#8302.

4. W. H. Gottschalk and G. A. Hedlund, Topological dynamics, Amer. Math. Colloq. Publ., vol. 36, Amer. Math. Soc., Providence, R.I., 1955. MR 17, 650.

5. S. Horowitz, Transition probabilities and contractions of $L_{\infty}, Z$. Wahrscheinlichkeitstheorie und Verw. Gebiete 24 (1972), 263-274.

6. M. Loève, Probability theory, 3rd ed., Van-Nostrand, Princeton, N.J., 1963. MR 34 \#3596.

7. J. Neveu, Mathematical foundations of the calculus of probability, Holden-Day, San Francisco, Calif., 1965. MR 33 \#6660.

8. K. Yosida and S. Kakutani, Operator theoretical treatment of Markoff's process and mean ergodic theorem, Ann. of Math. (2) 42 (1941), 188-228. MR 2, 230.

Department of Mathematics, Ohio State University, Columbus, Ohio 43210

Current address: Institute of Mathematics, Hebrew University, Jerusalem, Israel 